\title{
Analysis of the Risks of Development Injury Related to Surgical Positioning in a University Hospital of the State of Goiás/Brazil: An Observational-Epidemiological Study
}

\author{
Bárbara Almeida Lemes', Barbara Correia Neves Sabino',2, Carlos Eduardo Carvalho Sabino3, \\ Ana Clara Lenza Martins' ${ }^{1}$, Ana Letícia Neller Finta ${ }^{1}$, André Vinícius de Oliveira1, \\ Mariana Cordeiro Carvalho', Carolina Veras Mendes' ${ }^{1}$, Giulia Ugucioni Garbelini', \\ Lara Cândida de Sousa Machado ${ }^{1}$, Idiberto José Zotarelli Filho ${ }^{*}$
}

${ }^{1}$ Rio Verde University-UniRv, Rio Verde, Goiás, Brazil

${ }^{2}$ Faculty of Medical Sciences, State University of Campinas-UNICAMP, Campinas, Brazil

${ }^{3}$ Southwest Emergency Hospital-HURSO, Brazil

${ }^{4}$ Faceres Medical School of São José do Rio Preto/SP, Brazil

Email: *m.zotarelli@gmail.com

How to cite this paper: Lemes, B.A., Sabino, B.C.N., Sabino, C.E.C., Martins, A.C.L., Finta, A.L.N., de Oliveira, A.V., Carvalho, M.C., Mendes, C.V., Garbelini, G.U., de Sousa Machado, L.C. and Filho, I.J.Z. (2020) Analysis of the Risks of Development Injury Related to Surgical Positioning in a University Hospital of the State of Goiás/Brazil: An Observational-Epidemiological Study. Health, 12, 876-890.

https://doi.org/10.4236/health.2020.127065

Received: March 26, 2020

Accepted: July 26, 2020

Published: July 29, 2020

Copyright (อ 2020 by author(s) and Scientific Research Publishing Inc. This work is licensed under the Creative Commons Attribution International License (CC BY 4.0).

http://creativecommons.org/licenses/by/4.0/ (c) (i) Open Access

\begin{abstract}
Introduction: The perioperative nurse plays an important role throughout the surgical period, especially in the context of predicting the risks to which patients are exposed when entering a surgical center. These can be skin, muscle, tendon, and even burn injuries from the scalpel or chemicals. Objective: The aim of this study was to verify the degree of risk for the development of injuries resulting from surgical positioning in patients of a university hospital. Methods: This is cross-sectional, exploratory research with a quantitative approach conducted with 70 surgical patients in a Municipal University Hospital with data collection through a socioeconomic and health questionnaire and the Risk Assessment Scale for Development of Surgical Positioning Injuries (SISP). Statistical analyses included simple descriptive evaluation, correlations of variables using the Chi-square test and Pearson's coefficient with a significance level of $5 \%$. Results: The results found pointed to a population mainly composed of women (60\%), married, with an average age of 49 years, with hypertension and high-risk scores to develop injuries due to surgical positioning. There was also a positive and significant correlation between having hypertension and having a higher risk of developing lesions, with $\mathrm{R}^{2}=96.4 \%$ and $\mathrm{p}<0.05$. Conclusion: The results reinforce the need to implement protocols and the acquisition of materials aimed at reducing the risk of injuries related to surgical positioning in order to improve the quality of care offered and thus minimize the harm to users.
\end{abstract}




\section{Keywords}

Nursing, Pressure Injury, Operating Room

\section{Introduction}

Since ancient times, nursing plays an important role in the surgical field. Florence Nightingale participated in the preparation of the wounded soldiers who needed surgical intervention during the Crimean War (1853-1856), and at that time patient safety was already an integral part of their care due to the scenario of infection, bleeding and lack of materials. Gradually, Nursing ended up guaranteeing its space in the surgical sector, being a field that is increasingly expanding [1].

The perioperative nurse plays an important role throughout the surgical period. The first step is the reception of the patient and his family, with guidance on the aspects of the procedure and routines of the unit. Daily visits to clear doubts and analyze the patient's condition, checking vital signs, physical examination and prescribing individualized interventions should also be actions of nursing seeking comprehensive care. This promotes a good professional-patient relationship, as well as decreasing the individual's anxiety and transmitting greater security about care [2].

Despite numerous advances in Brazilian public health, the Unified Health System (SUS) still has a great shortage of material, human and managerial resources. This is explained by the high demand for services, a high number of surgeries, inadequate team size, management failure and lack of inputs. The combination of all these factors makes it difficult to plan prevention actions. This is due to the fact that Brazilian policies with a focus on prioritizing service quality were only developed from 2013 [3].

The National Patient Safety Program (PNSP) was established by the Ministry of Health in 2013 to focus on patient safety above any other premise, working to provide harm-free care. This ministerial program has six pillars, namely: 1) correctly identifying the patient; 2) improve communication between health professionals; 3) improve safety in prescribing and handling medications; 4) safe surgery at intervention site, patient and correct procedure; 5) sanitize hands to prevent infection and 6) reduce the risk of falling and pressure injury (PI) [4].

The second pillar of this program works with one of the most important tools of good care, effective communication, which in the context of the operating room is essential, taking into account the complexity of the procedures and the damage that improper performance can cause. True, accurate, and correctly passed information reduces errors, ultimately ensuring the patient's quality of life and proper recovery [4] [5].

The fourth pillar is specific for surgical interventions, as it recommends a pre-surgical conference on incision site, patient and procedure data, and prior 
testing of operating room equipment functionality. All data must be documented in medical records, including history and physical examination, signed a consent form and anesthetic induction and evaluation process [4].

The sixth and last pillar is strictly caring, which depends on a qualified intervention throughout the hospital stay. In the case of the risk of falls and IP, the professional should assess the patient's exposure to these two conditions and what can be done through validated scales, such as SISP. With the data collected from these, it is possible to reduce them through primarily simple interventions such as decubitus changes, skin hydration, aid and encouragement of ambulation when possible and the correct surgical positioning on the table, together with the use of devices, pressure reducers [4].

Regarding the PI, as to the definition they are recognized because they are injuries caused by the association between the factors pressure, sensitivity, skin microclimate, and tensile/shear forces. Its prevalence is higher in bony prominences but may arise as a result of improper use of medical materials and devices or due to incorrect positioning in both bed and operating tables. Classifications are divided into four stages according to the International Pressure Ulcer Classification System (NPUAP/EPUAP), taking into account the involvement of the skin and/or structures [6].

Stage 1 of IP are those where there is a flushing of the tissue, usually prominent, may be hot or cold and painful. It is characterized by being a healthy skin with erythema that does not whiten. Stage 2, on the other hand, has a bright and superficial appearance, with a pink tinge, and there is already a partial loss of skin with exposure to the dermis [7].

In stage 3 there is a total loss of skin structures, which eventually exposes the adipose tissue. In staged lesions 4 , tissue loss is similar to stage 3 , but there is exposure of structures (bones, tendons) and tissue loss. There are also nonstable lesions, which are those where there is necrotic tissue and also deep tissue injuries, caused by intense shear pressure. Medical device-related injuries and mucous membrane lesions are generated by the misuse of hospital equipment that should be used for therapeutic purposes [7].

Patients undergoing surgical procedures may still be exposed to other types of damage, such as tendon, muscle, chemical burns, and electric scalpel injuries. In the latter situation, most of the damage is caused by the incorrect placement of the dispersive plate, which may be justified by the inattention, haste, or unpreparedness of the professionals involved. All these situations are preventable through simple actions, such as correct positioning on the operating table, good preparation of the care team and the correct handling of available equipment [8].

Several injury prevention tools are available to add quality care and prevent physical and psychological harm to the patient. Operating table cushions, adhesives, covers, plates, and topical solutions are examples of materials that effectively accomplish this goal. They are costly devices, that is, they demand a great investment for their acquisition, but they are effective strategies for long-term 
planning and management, as they reduce the expenses with hospitalizations, the use of medications and fewer hours of nursing dedication, as well as preventing the institution suffer legal proceedings [9].

With regard to roofing, the market has a quality technological apparatus. There are prevention plaques that can be applied in the immediate preoperative period, and services for areas of bony prominence that will be in direct contact with the operating table or other medical devices. Thus, even before the patient begins his surgical procedure, he will be subjected to a safety intervention in order to reduce the risks of injuries related to surgical positioning [10].

These are covers that have a contact layer with silicone adhesive that minimizes pain, prevents skin damage and protects the wound. For each type, feature and location, there is an appropriate dressing, ranging from pressure injury prevention to treatment of chronic wounds, burns, surgical incisions, abrasions, and cuts. Materials of this quality reduce the frequency of changes, reduce the risk of surgical site infections and minimize costs to the institution [10].

Aiming at prioritizing patient safety and avoiding damage such as those previously discussed, injury risk assessment instruments have been developed, such as the Risk Assessment Scale for the Development of Injuries Due to Surgical Positioning (SISP). This includes items such as surgical position, time of surgery, age, comorbidities, and others, which in turn are relevant factors with regard to developing injuries. Its importance is based on the idea of providing nurses with early recognition of patients with higher risks, thus enabling the planning of effective and timely nursing actions [11].

The scale has served as the basis for research and protocol implementations, and manuals in health institutions. A large philanthropic hospital in the state of São Paulo has prepared a manual, which illustrates through images the implementation of the correct positioning and the risks involved in this process, once again highlighting the relevance that nursing has in the early identification of risks. The use and association of SISP with other instruments and with protective and interventionist measures incidentally reduces injuries arising from the assistance provided in the operating room [12].

Even in hospitals that lack the financial resources to purchase large numbers of prevention devices, with the implementation of specific protocols for IP care and nursing staff awareness, it is possible to reduce the incidence of iatrogenesis. This includes simple actions such as having pillows, cushions, and using covers such as hydrocolloid plates, as well as constantly performing scales such as Braden's and SISP's, which provide the necessary subsidies to provide excellent service [13].

Associated with all these technological apparatus of inputs, equipment, and scales, the continuing education of the operating room staff should be a continuous complement to the quality of care. The nurse in this sector, together with the permanent education team, has the role of ensuring and supervising the work of the teams, proposing conduct, instituting protocols and standard operating procedures (SOP) capable of providing instructions to professionals, identifying 
faults and correcting them. These actions can be accomplished through training and qualifications on relevant topics, such as equipment handling, patient approach, identification and minimization of surgical risks, making professionals aware of the importance of performing safe care practices [14].

Based on the relevance of this issue to institutions and health teams, the aim of this study was to verify the degree of risk for the development of perioperative injuries in surgical patients of a University Hospital by applying a validated scale, to SISP.

\section{Methods}

\subsection{Study Design}

This was an observational, cross-sectional, exploratory, descriptive study with a quantitative approach to the risk of injury development, socioeconomic and health factors of patients undergoing surgical procedures at a municipal university hospital in the city of Rio Verde, southwest of the state of Goiás (Table 1). A total of 70 patients were interviewed, prospectively according to the calculated sample size. STROBE rules followed (Strengthening the Reporting of Observational studies in Epidemiology https://www.strobe-statement.org/index.php?id= strobe-home) [15].

Table 1. Data relating to surgical procedures, 2019.

\begin{tabular}{|c|c|c|}
\hline \multirow{2}{*}{ Sample characterization variables } & \multicolumn{2}{|c|}{ Patients } \\
\hline & $\mathbf{N}$ & $\%$ \\
\hline \multicolumn{3}{|l|}{ Surgical Specialty } \\
\hline General & 32 & 46 \\
\hline Gynecology & 17 & 24 \\
\hline Orthopedics & 9 & 13 \\
\hline Proctology & 4 & 6 \\
\hline Plastic & 3 & 4 \\
\hline Pneumology & 2 & 3 \\
\hline Otorhinolaryngology & 1 & 2 \\
\hline Urology & 1 & 1 \\
\hline Vascular & 1 & 1 \\
\hline Total & 70 & 100 \\
\hline \multicolumn{3}{|l|}{ Surgery Character } \\
\hline Elective & 59 & 84 \\
\hline Urgency & 11 & 16 \\
\hline Total & 70 & 100 \\
\hline \multicolumn{3}{|l|}{ Size of Surgery } \\
\hline Small size & 33 & 47 \\
\hline Midsize & 33 & 47 \\
\hline Large & 4 & 6 \\
\hline Total & 70 & 100 \\
\hline
\end{tabular}




\subsection{Institution and Infrastructure}

This institution is located in a municipality in the Midwest region of Brazil, which currently has about 229,000 inhabitants. It is a medium-sized hospital with 100 beds, including surgical, clinical, complementary and pediatric beds. It was founded in 1989 by the governor of the time, Henrique Santillo, and the surgical unit established full operation only the following year, in 1990.

The institution's Surgical Center has 05 operating rooms and 01 post-anesthetic recovery room with 02 beds. The type of assistance provided is solely public, through the Unified Health System (SUS), with elective, urgent and emergency care, as well as laboratory and imaging diagnostic services.

The team consists of 103 physicians and 285 other health professionals, including nurses, physiotherapists, nutritionists, nursing technicians, psychologists, pharmacists, and occupational therapists. There are 16 specialties in this unit, including cardiology, general surgery, vascular, gastroenterology, gynecology, hematology, nephrology, pediatrics, infectology, and others.

\subsection{Participants}

Conscious individuals over 18 years of age who were present on the scale of surgical procedures in the given period of data collection, regardless of specialty and those who freely consented to participate in the study were included in the study. Those who, in the immediate preoperative period, presented any type of injury, decreased tissue perfusion, items that were verified in a brief preoperative physical examination, as well as polytraumatized patients and those who refused to sign the free and informed consent were excluded.

\subsection{Settings}

The recruitment took place at the Municipal University Hospital of Rio Verde (HMU) during December/2018 and January/2019, from 7 am to 5 pm. Individualized preoperative visits were performed in the operating room beds, in order to invite patients to participate in the research. Those who agreed received guidance, including reading and signing the consent form, as well as clarifying doubts. There was no refusal to participate by any patient. For data collection, two instruments were used: a socioeconomic and health characterization questionnaire, and a risk assessment instrument for injuries resulting from surgical positioning, the SISP scale.

The questionnaire was built by the researchers and addresses socio-demographic aspects such as gender, age, occupational status, education, marital status, monthly income and also health data such as comorbidities, previous surgeries, type and reason of surgery, waiting time for surgery, surgical specialty, character, and size of the surgery.

The SISP scale is a generic risk assessment instrument for the development of surgical positioning injuries that was developed by a Ph.D. in Nursing at the Medical-Surgical Nursing Center of the Federal University of Rio de Janeiro [11]. 
It is composed of seven items, including position type, anesthesia type, surgery time, support surface, limb position, comorbidities, and age. Each item has five sub-items that are scored from 1 to 5 , and its score includes from 7 to 35 points. Patients with scores above 20 are considered at high risk for injury development, and below 19 are considered at low risk [11].

\subsection{Ethical Aspects}

The right to choose to participate was preserved, and only those patients who consented to participate and signed the consent form were interviewed. The names of the participants were safeguarded, with only their initials and the name of the institution appearing in the questionnaire, thus preserving confidentiality and confidentiality. At the end of the research, all material was safely kept in the possession of the researcher where it will remain for the next 5 (five) years, according to Resolution 510/16 of the National Research Ethics Committee (CONEP).

This research was submitted to the Human Research Ethics Committee (CEP) of the University of Rio Verde (UniRV) and was approved with Opinion Number 2,974,979 and CAEE: 99609118.9.0000.5077.

\section{Statistical Analysis}

As for the statistical analysis of the data, the SISP score was calculated for each interviewed patient and the data were recorded in a spreadsheet together with those collected by the socioeconomic and health instrument. In addition to performing descriptive statistics (mean and standard deviation), we analyzed the variables investigated using the Statistical Package for Social Science (SPSS)-13.0 for Windows program, adopting a significance level of 5\% and for analysis of the variables. Correlations of data from both questionnaires were used Pearson correlation test and Chi-square test.

\section{Results and Discussion}

Seventy patients who were hospitalized for surgery were interviewed, of which 42 were female (60\%) and 28 male (40\%). Ages ranged from 21 to 86 years, and the average remained at 49 years with a standard deviation of 18.40 .

Regarding the respondents' education, there was a prevalence of incomplete elementary school (57\%), followed by complete high school (16\%), incomplete high school (11\%) and illiterate (6\%). The lowest percentage remained among those who had completed higher education (3\%).

When asked about their marital status, $49 \%$ of the sample said they were married, while $20 \%$ claimed consensual union without civil registration. The remaining participants were divided into single (17\%), widowed (10\%) and separated (6\%). Subsequent to this, they were asked whether they had children or not, with $93 \%$ of the affirmative answers. The average number of children remained at 2 , with a minimum of 0 and a maximum of 15 , according to data. 
Another aspect addressed by the questionnaire was the situation of the residence in which the patients lived, with three options of answers available: proper, which constituted $59 \%$ of the sample; rented with $37 \%$, and own financed with $4 \%$. Most of those who claimed to have paid housing still stressed living with their relatives (father, mother, grandparents), or even that the domicile would be given by them.

Participants were also questioned about their occupational status, with dominance of active people (34\%). Those who reported not having wage income and employment at the time of the interview reported being dependent (27\%), followed by retirees (21\%) and pensioners (4\%).

During the subsequent analysis of the data, it was found that the sample available for the topic "professional category" is relatively smaller, due to the inadequate answers given by respondents. The patients' professions were didactically divided into the subareas: services, agriculture, industry, housewives and unemployed. Regarding monthly income, the majority of patients (41\%) reported receiving only a minimum wage from their salaried work or even retirement.

Garcia et al., 2017 analyzed the socioeconomic characteristics of patients undergoing cataract surgery in a University Hospital of Minas Gerais, Brazil. The results corroborate those of this study, where among 148 patients analyzed, most were female, married, with incomplete elementary school and with family income between 2 minimum wages. These findings reinforce the premise that females are routinely the most predominantly submitted to surgical procedures in Brazil, which may be justified by the fact that women are the ones who most frequently seek disease prevention and screening actions, the most common ones. which in turn leads them to be diagnosed and treated more often in the health system [16].

Garcia et al., 2017 also analyzed the waiting factor for surgery, and in their study patients waited on average 3.5 to 8.1 months from consultation until the procedure. This shows the lack and the huge failure on the part of the Brazilian health system regarding the scheduling and care of patients since their role must be the resolution of problems and the reduction of harm to the individual. This delay may end up aggravating the user's pathological condition, thus leading to serious and even irreversible consequences [16].

A study conducted in 2017 at a Rio de Janeiro University Hospital assumed the objective of evaluating the epidemiological profile of patients treated at a pre-anesthetic evaluation outpatient clinic. The results obtained, similar to this research, showed that $50.1 \%$ of the patients studied had hypertension, followed by diabetes mellitus, lung disease, and dyslipidemia. This reinforces epidemiological data that point out that Hypertension is the most prevalent and prevalent chronic disease in the Brazilian population today, being a serious public health problem and requiring qualified interventions [17].

The predominant surgical specialty was general surgery (46\%), as in a study conducted in 2014 that had as its field a public university hospital in the state of 
Minas Gerais. The results of this research were that $29 \%$ of the 351 patients analyzed underwent abdominal surgeries, that is, in the "general" type. The explanation for this may be that this medical field has a wide variety of surgical procedures and includes a greater number of organs in the human body, which are those located between the diaphragm and the pelvis, such as the stomach and intestine [18].

Supporting surfaces ensure pressure relief and preserve the client's skin integrity and considerably reduce the risks to which the patient is exposed, mitigating muscle, skin and joint damage. These interventions are simple and bring great benefits to care, ensuring the performance of a safe and effective procedure, such intervention was not observed in the present research, which in turn may justify the high scores obtained by SISP. Oliveira et al., 2018 found in their study that the adoption of the strategies mentioned above by the health team was relevant to obtain lower SISP scores in $62.2 \%$ of surgeries followed during their research [19].

Oliveira et al., 2018 also evaluated the risk of developing surgical injuries by positioning through the SISP in a public hospital of medium and high complexity in the state of Piauí, however he found that $68.9 \%$ of patients had low risk for developing injuries, However, despite the fact that this is a research where there was a predominance of small and medium-sized procedures, it is explained by the lack of measures in the unit studied perioperative protective measures [19].

Peixoto et al., 2019 evaluated and classified patients according to the Risk Assessment Scale for Perioperative Pressure Injuries, to verify the association between sociodemographic and clinical variables and the risk score, and identify the occurrence of pressure injuries due to surgical positioning [20]. Most participants had a high risk of developing perioperative decubitus ulcers. Female gender, elderly group, and altered body mass index were significant factors for the increased risk. The Risk Assessment Scale for Perioperative Pressure Injuries allows early identification of the risk of injury, supporting the adoption of preventive strategies to ensure the quality of perioperative care [21].

Regarding the data collected regarding health conditions, as for comorbidities, 41 patients (59\%) reported having at least one non-transmissible chronic disease (NCD), which were mostly female with the prevalence of Systemic Arterial Hypertension (HAS). A positive correlation was observed between the variables age and comorbidity, and people over 38 years had a higher incidence of NCDs, this was confirmed by the Pearson correlation test with a significance level of 0.01 .

When asked about previous surgeries, $76 \%$ of participants said they had undergone other surgical procedures at some time in their lives. The most frequently reported were cesarean section, tubal ligation, herniorrhaphy, hysterectomy, appendectomy, and fracture correction. Regarding the type, the surgeries were divided based on the body system involved, with a predominance of those involving the digestive tract (49\%), followed by the genitourinary tract (27\%) and respiratory tract $(13 \%)$. 
The reason for the surgeries was mostly related to the procedure itself, such as acute cholelithiasis, inguinal, epigastric and/or umbilical hernias, desire for definitive contraception, weakening of the pelvic floor muscles, falls from his own height and car accidents. The average waiting time for the surgical procedure was around six months (180 days), with a maximum of two years (730 days) for inguinal herniorrhaphy surgery and a minimum of one day for appendectomy and amputation surgeries. The period between diagnosis and surgical indication until the procedure was always taken into consideration.

Regarding the SISP scale, the results obtained were significant (Table 2 and Table 3). After counting the scores, most respondents fit the high-risk category (61\%) (Figure 1) for developing IP (score over 20), where the prevalence in females stood out (58), 1\%) and with hypertension. The highest score remained at 29 points and the lowest at 12 . It is noteworthy that the topic "support surface" was an aggravating factor during the application of the instrument because the operating tables of all operating rooms were composed of an inadequate material which offers low comfort to patients and consequently a higher risk of injury development.

During the data collection of the HMU of Rio Verde-GO, there were no attitudes of the health team aimed at preventing injuries resulting from surgical positioning and there were no types of pressure reducing devices in the operating rooms.

Regarding the risk of injury identified in the present study, the statistical analysis obtained a significant correlation (Pearson correlation at level 0.01) between having a hypertension-type NCD and having a high score for the development of injuries related to surgical positioning. This finding points to the need to develop more carefully preventive actions for surgical patients with hypertension.

Corroborating with the guidelines of the SISP scale, the present study confirmed by statistical correlation tests that the older the patient is, the greater the risk of developing injuries, as well as the longer the surgery and the more sedated the greater the risk, with $\mathrm{p}<0.01$. It was also found that with increasing age the patient is more likely to be exposed to longer surgical times and consequently to greater risks.

\section{SISP SCORES}

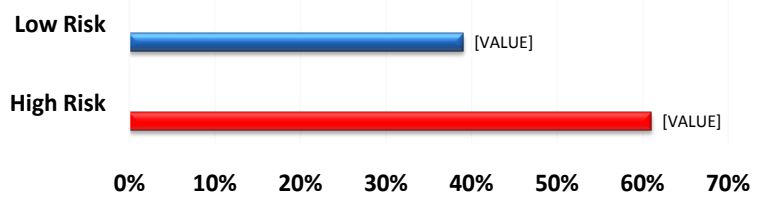

Figure 1. SISP scores. 
Table 2. Percentage of SISP score in relation to age.

\begin{tabular}{|c|c|c|c|}
\hline \multirow{2}{*}{ Age } & \multicolumn{2}{|c|}{ Score SISP } & \multirow{2}{*}{ Total } \\
\hline & Low Risk & High Risk & \\
\hline 21.00 & $3.7 \%$ & & $1.4 \%$ \\
\hline 22.00 & $3.7 \%$ & $2.3 \%$ & $2.9 \%$ \\
\hline 23.00 & & $2.3 \%$ & $1.4 \%$ \\
\hline 24.00 & $3.7 \%$ & & $1.4 \%$ \\
\hline 26.00 & $7.4 \%$ & & $2.9 \%$ \\
\hline 28.00 & $7.4 \%$ & $2.3 \%$ & $4.3 \%$ \\
\hline 29.00 & $3.7 \%$ & & $1.4 \%$ \\
\hline 30.00 & $7.4 \%$ & & $2.9 \%$ \\
\hline 32.00 & $3.7 \%$ & & $1.4 \%$ \\
\hline 33.00 & $7.4 \%$ & & $2.9 \%$ \\
\hline 35.00 & $3.7 \%$ & & $1.4 \%$ \\
\hline 36.00 & $3.7 \%$ & $7.0 \%$ & $5.7 \%$ \\
\hline 37.00 & $3.7 \%$ & & $1.4 \%$ \\
\hline 38.00 & $11.1 \%$ & $2.3 \%$ & $5.7 \%$ \\
\hline 39.00 & & $2.3 \%$ & $1.4 \%$ \\
\hline 40.00 & & $2.3 \%$ & $1.4 \%$ \\
\hline 42.00 & & $4.7 \%$ & $2.9 \%$ \\
\hline 44.00 & & $2.3 \%$ & $1.4 \%$ \\
\hline 45.00 & & $2.3 \%$ & $1.4 \%$ \\
\hline 46.00 & $3.7 \%$ & & $1.4 \%$ \\
\hline 47.00 & $3.7 \%$ & $4.7 \%$ & $4.3 \%$ \\
\hline 48.00 & & $2.3 \%$ & $1.4 \%$ \\
\hline 49.00 & & $2.3 \%$ & $1.4 \%$ \\
\hline 51.00 & $3.7 \%$ & & $1.4 \%$ \\
\hline 54.00 & $7.4 \%$ & & $2.9 \%$ \\
\hline 55.00 & & $7.0 \%$ & $4.3 \%$ \\
\hline 56.00 & $3.7 \%$ & $7.0 \%$ & $5.7 \%$ \\
\hline 58.00 & & $2.3 \%$ & $1.4 \%$ \\
\hline 59.00 & & $2.3 \%$ & $1.4 \%$ \\
\hline 61.00 & & $2.3 \%$ & $1.4 \%$ \\
\hline 63.00 & & $2.3 \%$ & $1.4 \%$ \\
\hline 64.00 & & $7.0 \%$ & $4.3 \%$ \\
\hline 68.00 & $3.7 \%$ & $2.3 \%$ & $2.9 \%$ \\
\hline 69.00 & & $2.3 \%$ & $1.4 \%$ \\
\hline 70.00 & & $4.7 \%$ & $2.9 \%$ \\
\hline
\end{tabular}




\section{Continued}

\begin{tabular}{|c|c|c|c|}
\hline 72.00 & & $2.3 \%$ & $1.4 \%$ \\
\hline 76.00 & & $2.3 \%$ & $1.4 \%$ \\
\hline 77.00 & & $2.3 \%$ & $1.4 \%$ \\
\hline 81.00 & & $4.7 \%$ & $2.9 \%$ \\
\hline 82.00 & & $2.3 \%$ & $1.4 \%$ \\
\hline 83.00 & $3.7 \%$ & & $1.4 \%$ \\
\hline 84.00 & & $4.7 \%$ & $2.9 \%$ \\
\hline 86.00 & & $2.3 \%$ & $1.4 \%$ \\
\hline & $100.0 \%$ & $100.0 \%$ & $100.0 \%$ \\
\hline
\end{tabular}

Table 3. Percentage of SISP score in relation to gender.

\begin{tabular}{|c|c|c|c|c|}
\hline & & \multicolumn{2}{|c|}{ Score SISP } & \multirow{2}{*}{ Total } \\
\hline & & Low Risk & High Risk & \\
\hline \multirow{2}{*}{ Gender } & Male & $37.0 \%$ & $41.9 \%$ & $40.0 \%$ \\
\hline & Female & $63.0 \%$ & $58.1 \%$ & $60.0 \%$ \\
\hline \multicolumn{2}{|c|}{ Total } & $100.0 \%$ & $100.0 \%$ & $100.0 \%$ \\
\hline
\end{tabular}

The analysis of data regarding the types of surgeries indicated that the majority of musculoskeletal appliances were performed in males (55.6\%), while in females there was a predominance of those involving the genitourinary tract (94.7\%). This fact led, due to the fact that surgical positioning is predominantly lithotomic, with higher high-risk scores for the development of lesions occur in women in the present study.

Researching situations that involve patient safety requires evaluating factors intrinsic to the health of these subjects, such as the existence of comorbidities and extrinsic factors such as the disposition and existence of materials, inputs and qualified and adequate human resources to provide holistic and safe care. After evaluating, measuring and discussing these factors through the present research, it is essential that interdisciplinary efforts capable of changing the reality are found, in which the majority of patients submitted to surgeries at the Municipal University Hospital have high risks to develop injuries related to surgical positioning.

This scenario should, in turn, be presented to raise awareness among the multidisciplinary health team and also the institution itself, as it is necessary that when patients with the identified risk characteristics (women, over 38 years old and with hypertension) enter into the hospital to perform surgeries, receive more qualified and directed attention, in order to mitigate the risk to which they are exposed and avoid further damage that may interfere with the health of these individuals.

It is known that the fact that the institution is public and has limited funds from SUS makes it difficult to obtain effective instruments for the prevention of 
injuries in the perioperative period, however, it is possible to carry out continuing education campaigns, implementation of protocols using the SISP, and even budget planning that enables the purchase of materials to equip the unit's operating room.

The idea is to provide nurses and their teams with ways of early recognition of patients who are more likely to develop injuries and also to provide the necessary resources for the planning and execution of effective and timely nursing actions, especially with regard to risk prevention related to surgical positioning.

\section{Conclusion}

The present study has provided important evidence related to the risk of developing injuries resulting from surgical positioning. However, for the generalization of these results, further research is needed to evaluate the correlation with other factors possibly associated with the occurrence of pressure injury caused by surgical positionings, such as Body Mass Index (BMI), body temperature and intraoperative blood pressure, and plasma albumin levels. It is suggested to perform a longitudinal study with an extended follow-up period for patients in the postoperative period in order to verify the amount of those who obtained a high-risk score and who actually developed lesions.

\section{Conflicts of Interest}

The authors declare no conflicts of interest regarding the publication of this paper.

\section{Funding}

We would like to thank financial support of Rio Verde University-UniRv, Rio Verde, Goiás, Brazil.

\section{Conflicts of Interest}

The authors declare no conflicts of interest regarding the publication of this paper.

\section{References}

[1] Santos, R., et al. (2018) The Role of Nurses in the Surgical Center. GEP NEWS, Maceió, 9-15.

[2] Sena, A.C., et al. (2017) Collective Construction of an Immediate Preoperative Nursing Care Instrument for Patients. Revista Baiana de Enfermagem, Florianópolis, 10.

[3] Capucho, H.C. and Cassiani, S.H.B. (2013) Need to Implement National Patient Safety Program in Brazil. Revista de Saúde Pública, 47, 791-798. https://doi.org/10.1590/S0034-8910.2013047004402

[4] Brasil, Ministério da saúde, ANVISA, Fundação Oswaldo Cruz (2014) Documento de referência para o Programa Nacional de Segurança do Paciente. Brasília, 40.

[5] Borges, F., et al. (2016) Degree of Patient Safety Culture in the Perception of the Multiprofessional Hospital Team. Revista Varia Scientia-Ciências da Saúde, Paraná, 12. 
[6] Vasconcelos, J.M.B. and Caliri, M.H.L. (2017) Nursing Actions before and after a Protocol for Preventing Pressure Injury in Intensive Care. Escola Anna Nery, 21, e20170001.

[7] SOBEST/SOBENDE (2016) Pressure Injury Classification-NPUAP Consensus-Culturally Adapted for Brazil. http://www.sobest.org.br/textod/35

[8] Bisinotto, F.M.B., et al. (2017) Electrosurgery-Related Burns-Report of Two Cases. Revista Brasileira de Anestesiologia, 67, 527-534. https://doi.org/10.1016/j.bjane.2015.08.018

[9] Galvão, N.S., Serique, M.A.B., de Gouveia Santos, V.L.C. and Nogueira, P.C. (2017) Knowledge of the Nursing Team on Pressure Ulcer Prevention. Revista Brasileira de Enfermagem, 70, 294-300. https://doi.org/10.1590/0034-7167-2016-0063

[10] Mölnlycke (2018). https://www.molnlycke.com/about-us/molnlycke-in-numbers

[11] Lopes, C.M., Haas, V.J., Dantas, R.A., Oliveira, C.G. and Galvão, C.M. (2016) Assessment Scale of Risk for Surgical Positioning Injuries. Revista Latino-Americana de Enfermagem, 24, e2704. https://doi.org/10.1590/1518-8345.0644.2704

[12] Sousa, C.S., Bispo, D.M. and Acunã, A.A. (2018) Creation of a Manual for Surgical Positioning: Experience Report. São Paulo: Revista SOBECC, 23, 169-175. https://doi.org/10.5327/Z1414-4425201800030009

[13] Stuque, A., et al. (2017) Protocol for Prevention of Pressure Ulcer. Revista da Rede de Enfermagem do Nordeste, 18, 272-282. https://doi.org/10.15253/2175-6783.2017000200018

[14] Ribeiro, M.B. (2017) A Continuing Education in Nursing Training at the Surgical Center: An Integrative Review. São Paulo: Revista SOBECC, 22, 98-105. https://doi.org/10.5327/Z1414-4425201700020007

[15] Elm, E.V., Altman, D.G., Egger, M., et al. (2007) The Strengthening the Reporting of Observational Studies in Epidemiology (STROBE) Statement: Guidelines for Reporting Observational Studies. PLOS Medicine, 4, 296. https://doi.org/10.1371/journal.pmed.0040296

[16] Garcia, O.D., Gomes Novais, S.S., Emboaba, S.A. and Karina, P. (2017) Assessment of Socioeconomic Profile, Vocational Training and Health Status of People with Visual Impairment. Revista Brasileira de Oftalmologia, 76, 255-258. http://www.scielo.br/scielo.php?script=sci_arttext\&pid=S0034-72802017000500255 \&lng=en https://doi.org/10.5935/0034-7280.20170053

[17] Loureiro, S.M., de Oliveira, N.C. and Carlos, I.A. (2017) Epidemiological Profile of Patients Treated at the Pre-Anesthetic Evaluation Outpatient Clinic of a University Hospital. The Revista Brasileira de Anestesiologia, 67, 457-467. http://www.scielo.br/scielo.php?script=sci_arttext\&pid=S0034-70942017000500457 \&lng=en https://doi.org/10.1016/j.bjane.2017.04.006

[18] Cunha, P.C.B., Elizabeth, B., da Silva, P.P., José, H.V. and Helena, B.M. (2015) Occurrence and Factors Associated with Intraoperative Hypothermia of Elective Abdominal Surgery. Acta Paulista de Enfermagem, 28, 475-481.

http://www.scielo.br/scielo.php?script=sci_arttext\&pid=S0103-21002015000500475 \&lng=en

[19] Hmbs, O., Amjf, S., Mza, M., Emlr, A. and Grf, S. (2019) Risk Assessment for the Development of Perioperative Injuries Resulting from Surgical Positioning. The Revista Gaúcha de Enfermagem, 40, e20180114. 
[20] Peixoto, C.A., Ferreira, M.B.G., Felix, M.M.D.S., Pires, P.D.S., Barichello, E. and Barbosa, M.H. (2019) Risk Assessment for Perioperative Pressure Injuries. Revista Latino-Americana de Enfermagem, 27, e3117. https://doi.org/10.1590/1518-8345.2677-3117

[21] Coyer, F., Clark, M., Slattery, P., Thomas, P., McNamara, G., Edwards, C., Ingleman, J., Stephenson, J. and Ousey, K. (2017) Exploring Pressures, Tissue Reperfusion and Body Positioning: A Pilot Evaluation. Journal of Wound Care, 26, 583-592. https://doi.org/10.12968/jowc.2017.26.10.583 